\title{
Standardization of the PQRST Waveform and Analysis of Arrhythmias in the Fetus Using Vector Magnetocardiography
}

\author{
HITOSHI HORIGOME, KUNIOMI OGATA, AKIHIKO KANDORI, TSUYOSHI MIYASHITA, MIHO TAKAHASHI-IGARI, \\ YANG-JEN CHEN, HIROMI HAMADA, AND KEIJI TSUKADA
}

\author{
Department of Pediatrics [H. Ho., M.T.-I.] and Department of Obstetrics and Gynecology [Y.-J.C., H. Ha.], University of Tsukuba, \\ Tsukuba 305-8575, Japan; Central Research Laboratory [K.O., A.K., T.M.], Hitachi Ltd., Kokubunji 185-8601 Tokyo, Japan; Department \\ of Electrical and Electronic Engineering [K.T.], Okayama University, Okayama 700-8530, Japan
}

\begin{abstract}
Fetal magnetocardiography (fMCG) is useful for analysis of fetal cardiac events. However, fetal presentation and movement affect the fMCG waveform, making it difficult to standardize the waveform. The aim of this study was to investigate whether the use of vector magnetometers can compensate for these limitations. We studied 59 fetuses (gestational age, 22-40 wk, median, 32), including 41 with uncomplicated pregnancies and 18 with fetal cardiac disease. fMCG was recorded twice in each case, and the two waveforms were compared with each other in uncomplicated subjects to investigate the effects of fetal presentation. The superconducting quantum interference device (SQUID) system used in this study was a 12-channel vector magnetometer, by which the three components of the magnetic field (Bx, By, Bz) could be detected simultaneously at four recording points. By constructing the three components, a composite waveform (Bxyz) was obtained. The configuration of the composite waveforms was similar among normal fetuses always with positive polarity, independent of fetal presentation and movement. The difference in the time intervals (PR, QRS, and ventricular activation time [VAT]) between the first and second measurements was minimal in the composite waveforms (Bxyz) compared with that in each channel (Bx, By, Bz). Even before signal averaging, waveforms with high time resolution were recorded in at least one of the three components, making it possible to analyze fetal arrhythmias precisely. Our results indicate that vector magnetocardiography is potentially useful for standardization of the fMCG waveforms and to provide a more complete and accurate analysis of fetal arrhythmias.
\end{abstract} (Pediatr Res 59: 121-125, 2006)

fMCG has gathered increasing interest in recent years, particularly its potential clinical application (1-5). fMCG has an advantage over fetal electrocardiography recorded on the maternal abdomen in detecting fetal heart signals because magnetic fields are far less insulated by vernix caseosa and less interfered by maternal signals (6). fMCG may also be advantageous to fetal echocardiography for precise analysis of arrhythmias because it reflects the electric not mechanical properties of the heart (3), although the latter is a more prevalent and valuable method with relevant time resolution. Several studies have already reported the usefulness of fMCG in the diagnosis and management of fetal arrhythmias $(5,7)$. Since fMCG provides detailed diagnosis of fetal arrhythmias,

Received February 22, 2005; accepted July 26, 2005.

Correspondence: Hitoshi Horigome, M.D., Department of Pediatrics, Institute of Clinical Medicine, University of Tsukuba, 1-1-1 Tennodai, Tsukuba 305-8575, Japan; e-mail: hhorigom@md.tsukuba.ac.jp

DOI: $10.1203 / 01 . p d r .0000190578 .81426 . f c$ it is feasible to administer antiarrhythmic agents transplacentally based on accurate fMCG-based diagnosis $(5,7,8)$.

One disadvantage of the fMCG waveforms is their tendency to be largely affected by fetal orientation and movement, making it difficult to compare the waveforms among fetuses in various presentations. fMCG waveforms even fluctuate during measurement in the same fetus. Furthermore, fMCG data somewhat vary depending on the superconducting quantum interference device (SQUID) system used and the protocol of data acquisition (9). Nevertheless, the magnetic field is a vector and three components $(\mathrm{Bx}, \mathrm{By}, \mathrm{Bz})$ can be measured individually and composed. The aims of this study were to determine whether we could obtain conformable waveforms with high resolution independent of fetal orientation by measuring all magnetic components simultaneously and composing them, as well as investigating the usefulness of vector magnetometers for compensation of the aforementioned limitations.

\section{METHODS}

Study population. We studied 59 fetuses (gestational age, 22-40 wk, median, 32), including 41 with uncomplicated pregnancy and 18 with fetal cardiac disease (11 with arrhythmia and seven with structural abnormalities). The study protocol was approved by the Human Ethics Review Committee of the Hospital, and a signed consent form was obtained from parents before commencement of the study.

Instrumentation and measurements. A 12-channel SQUID system housed in a magnetically shielded room was used in this study. The sensor array of the vector gradiometers contained the $x, y$, and $z$ axis pick-up coils at $2 \times 2$ points with an interval of $30 \mathrm{~mm}$, making the covering area $9 \mathrm{~cm}^{2}$. The baseline distance of the gradiometer was $60 \mathrm{~mm}$ for each axis. The three pickup coils are allocated perpendicularly to each other, allowing the simultaneous detection of the three components of the magnetic field (Bx, By, Bz). The pickup coils for detecting $\mathrm{Bz}$ are of the round type with a diameter of 20 $\mathrm{mm}$, while those for $\mathrm{Bx}$ and $\mathrm{By}$ are rectangular type $(20 \times 16 \mathrm{~mm})$. The magnetic flux resolution of the gradiometer was less than $10 \mathrm{fT} / \sqrt{ } \mathrm{Hz}$. By constructing three components using the following formula, a composite waveform (Bxyz) was obtained (10): $\mathrm{Bxyz}=\sqrt{ }\left(\mathrm{Bx}^{2}+\mathrm{By}^{2}+\mathrm{Bz}^{2}\right)$.

fMCG was recorded by approaching the sensor array to the maternal abdominal surface. The cryostat can be tilted up to 30 degrees to adjust the sensor position as close to the fetal heart as possible on the curved maternal abdominal surface based on fetal echocardiographic findings. Signal sampling was performed at a sampling rate of $1000 \mathrm{~Hz}$ for at least $2 \mathrm{~min}$, and the signals

Abbreviations: fMCG, fetal magnetocardiography; SQUID, superconducting quantum interference devise; VAT, ventricular activation time 

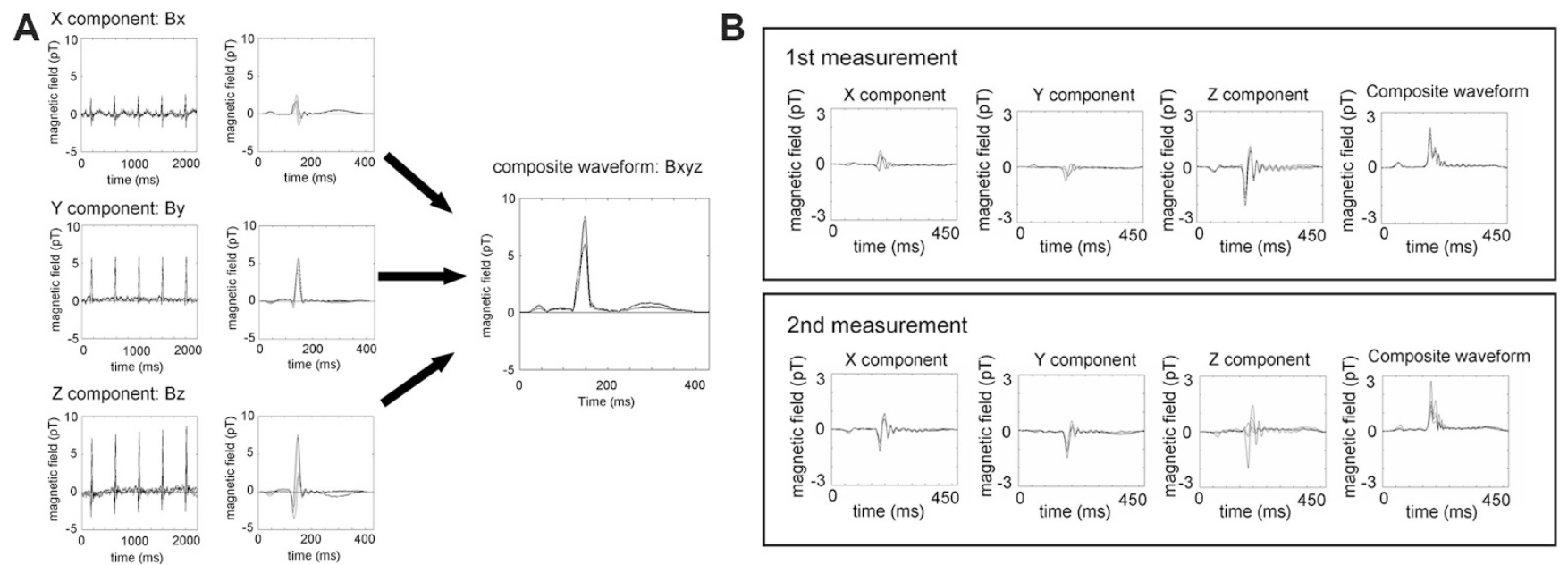

Figure 1. Vector magnetocardiograms. (A) Vector magnetometer components before (left column) and after (middle column) signal averaging triggered by the $\mathrm{R}$ peak. By constructing the three components of the magnetic fields (Bx, By, Bz), high time resolution PQRST waveforms (Bxyz) are obtained (right). The polarity of the composite waveforms is always positive, independent of fetal orientation and movement. (B) The waveforms of each component of the magnetic field (Bx, By, Bz) at the first measurement (top) are different from those at the second measurement (bottom). However, the composite waveforms obtained by constructing the three components (Bxyz) show similar configuration between the first and second measurements.

were then passed through a $0.1-$ to $100-\mathrm{Hz}$ and a $50-\mathrm{Hz}$ power-line filter. fMCG was recorded twice at different intervals (range, 2-48 h; median, $24 \mathrm{~h}$ ) in each case to compare the waveforms with each other and investigate the effects of fetal movement.

Two-dimensional fetal echocardiography was performed in each case using the SONOS-5500 system (Philips, Andover, MA) for diagnosis and exclusion of structural heart disease as well as for determination of fetal presentation immediately before the first and second fMCG measurements.

Data analysis. The success rate of recording $\mathrm{p}, \mathrm{QRS}$, and $\mathrm{T}$ waves was assessed for each component (Bx, By, Bz) before signal averaging. That for $\mathrm{p}$ and $\mathrm{T}$ waves after signal averaging triggered by the QRS peak and composition of the three components (Bxyz) was also assessed. The values of $\mathrm{PR}, \mathrm{QRS}$, and QT intervals were measured manually by one author who was skilled in this analysis after signal averaging followed by composition, and correlations of the intervals with the gestational age were investigated in uncomplicated fetuses. Differences in the values of PR and QRS intervals and VAT between the first and second measurements were also evaluated for uncomplicated subjects, and those differences were compared with each other among Bx, By, Bz and Bxyz. VAT was defined as the time from the onset of QRS complex to the positive peak. Fetal arrhythmias were analyzed without signal averaging of the waveform on tracings with distinctly recognizable $p$ waves among 12 channels.

Statistical analysis. The correlation of the time intervals with gestational age was analyzed using simple linear regression. The absolute values of the difference in milliseconds between the first and the second measurements were compared among $\mathrm{Bx}, \mathrm{By}, \mathrm{Bz}$ and $\mathrm{Bxyz}$ using one-way analysis of variance (ANOVA). Data are expressed as mean \pm SD. A $p$ value $<0.05$ was considered significant.

\section{RESULTS}

Ultrasonography revealed that all fetuses were in longitudinal position. The fetal presentation at the first fMCG mea-

\section{Normal fetuses}
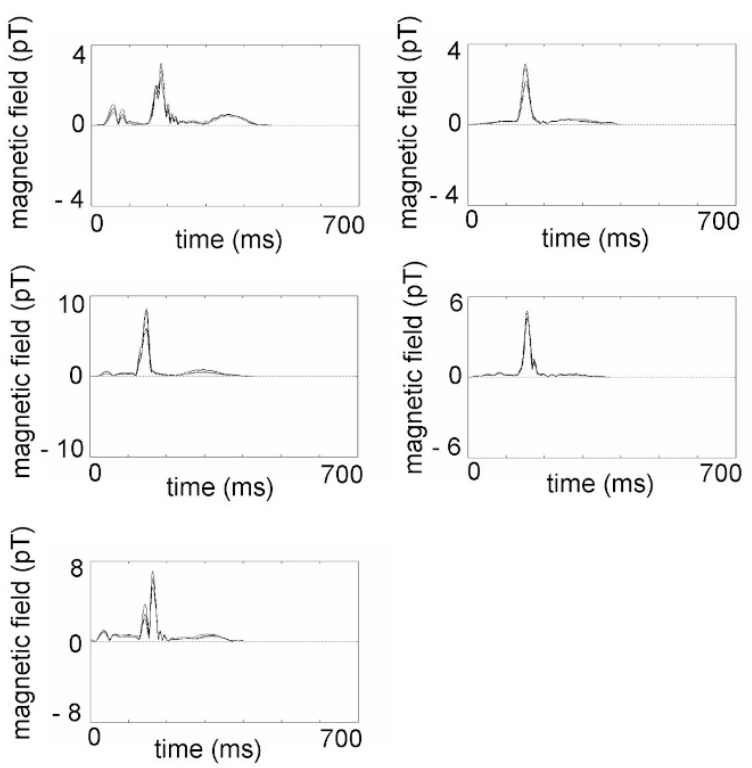

\section{Ventricular hypertrophy}
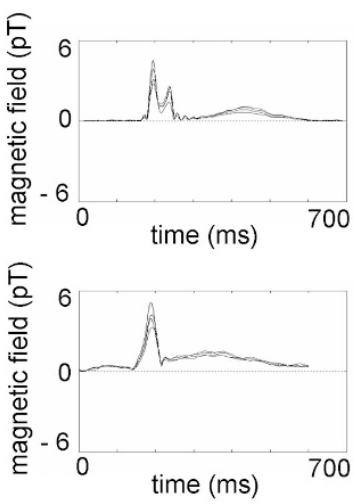

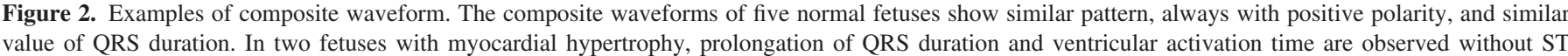
segment depression. 

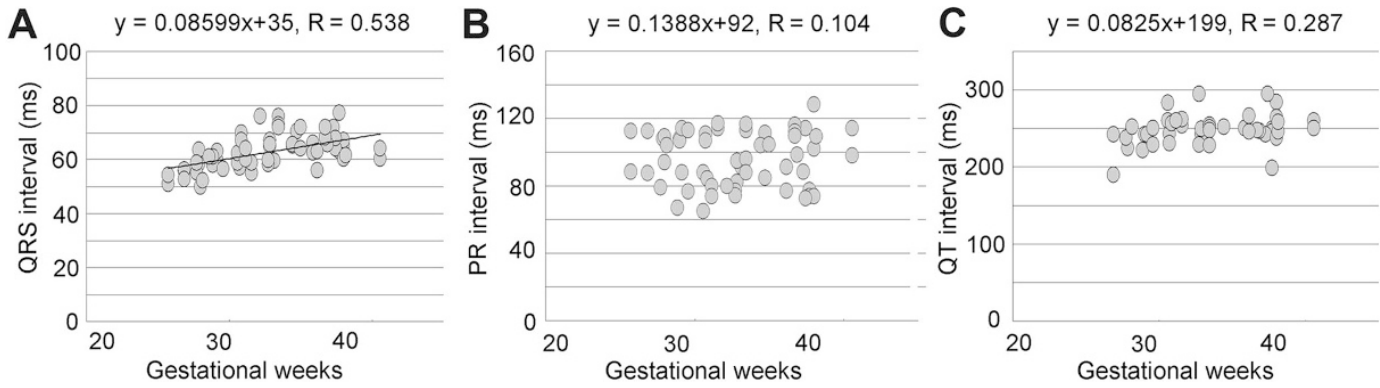

Figure 3. Relations between gestational age and cardiac time intervals. QRS interval shows positive correlation with gestational age (A), but PR (B) and QT (C) intervals are rather constant without significant correlation.

surement was vertex in 53 fetuses (90\% of all fetuses); 30 of them were in the left occiput position and 23 in the right. Six fetuses $(10 \%)$ were in breech presentation. Eighteen fetuses (30\% of all) moved after the first measurement and a significant change in the presentation was noted in the second measurement (from right/left occiput to left/right occiput in 11 cases, from breech to vertex in five cases, from vertex to breech in two cases). Based on the fMCG polarity change during the recording, we concluded that small fetal movements occur frequently in many fetuses but could not be determined correctly because ultrasonography could not be applied during fMCG measurement. The detection rate before signal averaging for QRS was 100\% in each channel and those for $\mathrm{p}$ and $\mathrm{T}$ waves were $91 \%$ and $53 \%$ for $\mathrm{Bx}, 75 \%$ and $56 \%$ for $\mathrm{By}$, and $94 \%$ and $75 \%$ for $\mathrm{Bz}$, respectively. Thus, the detection rates of $\mathrm{Bz}$ for both $\mathrm{p}$ and $\mathrm{T}$ waves were higher than the others. After signal averaging and composition of the three components (Bxyz), the detection rate for $\mathrm{p}$ and $\mathrm{T}$ waves increased to $97 \%$ and $78 \%$, respectively. Due to the lowamplitude nature of the $\mathrm{T}$ wave in the fetus, $22 \%$ of the subjects showed ambiguous onset or endpoints of the $T$ waves even though a $\mathrm{T}$ wave appeared, and those cases were excluded from further analysis. The detection rates for $\mathrm{p}$ and $\mathrm{T}$ waves varied significantly with gestational age; those for fetuses at full term were over $95 \%$.

By constructing the three components of the magnetic fields (Bx, By, Bz), high time resolution PQRST waveforms were obtained (Fig. 1). The composite waveforms showed similar pattern among normal fetuses, always with positive polarity. QRS waveforms with abnormal shape and prolonged duration were recognized in the fetuses with myocardial hypertrophy. Examples of the composite waveform in five normal fetuses and two fetuses with myocardial hypertrophy are presented in Fig. 2.

The QRS intervals in normal fetuses ranged from 50 to 85 ms $(64 \pm 6$, mean $\pm \mathrm{SD})$, and correlated with gestational age.

Table 1. Differences between values of the first and second measurements

\begin{tabular}{lccccc}
\hline Parameter & $\mathrm{Bx}$ & $\mathrm{By}$ & $\mathrm{Bz}$ & $\mathrm{Bxyz}$ & $p$ value \\
\hline PR (ms) & $5.6 \pm 5.3$ & $6.3 \pm 6.6$ & $7.2 \pm 4.2$ & $2.9 \pm 2.6$ & 0.048 \\
QRS (ms) & $5.1 \pm 3.6$ & $4.0 \pm 4.5$ & $3.3 \pm 3.2$ & $2.3 \pm 1.7$ & 0.022 \\
VAT (ms) & $3.8 \pm 2.9$ & $4.1 \pm 3.2$ & $3.3 \pm 2.4$ & $2.6 \pm 1.7$ & 0.043 \\
\hline
\end{tabular}

Values are expressed as mean \pm SD. Values are compared among Bx, By, $\mathrm{Bz}$ and Bxyz using one-way ANOVA.
The PR interval ranged from 67 to $128 \mathrm{~ms}(97 \pm 14 \mathrm{~ms})$ and QT interval ranged from 189 to $295 \mathrm{~ms}(248 \pm 13 \mathrm{~ms})$, both being rather constant during the period of concern (Fig. 3). Differences in the time interval values (PR, QRS, and VAT) between the first and second measurements are presented in Table 1. The differences were significantly smaller in Bxyz compared with each channel before composition (Bx, By, Bz).

Fetal arrhythmias were analyzed on the tracing with distinctly recognizable $p$ waves and the highest amplitude among 12 channels before composing three magnetic components. Typically, the resolution of at least one of the tracings was satisfactory for analysis, and detailed investigation of fetal arrhythmia was possible (Fig. 4). fMCG diagnoses of the fetuses with arrhythmias or structural heart disease are presented in Table 2. For example, in a fetus with a gestational age of $30 \mathrm{wk}$, congenital complete atrioventricular block was complicated by short run type ventricular rhythm (Fig. 5). The $\mathrm{RP}^{\prime}$ and $\mathrm{P}^{\prime} \mathrm{R}$ intervals were confirmed to be precise by comparing them with those determined by electrocardiography after birth in fetuses with supraventricular tachycardia. Fur-
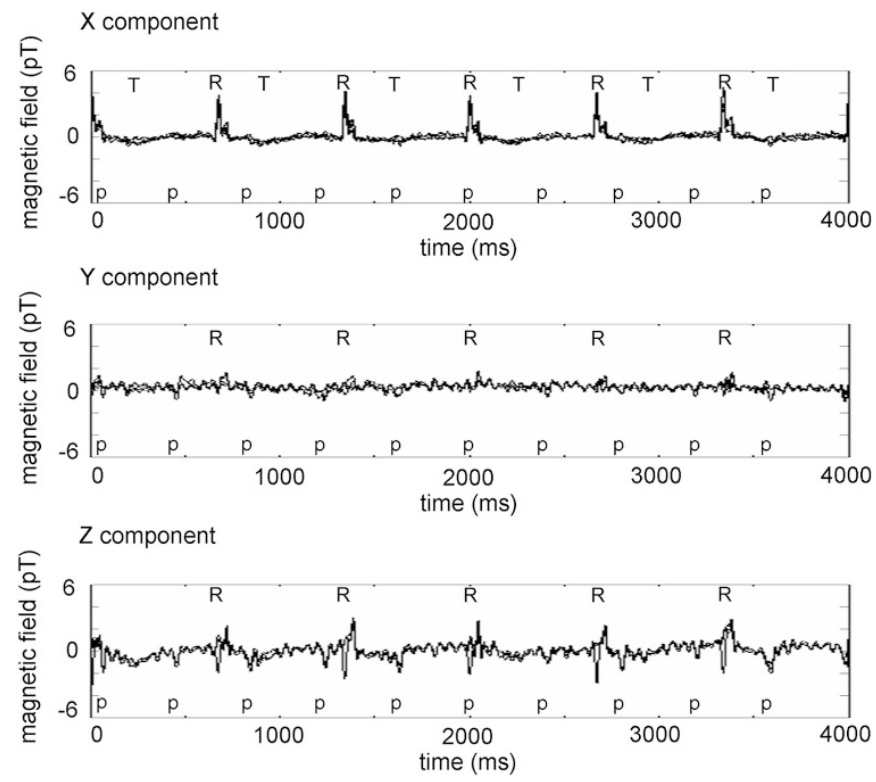

Figure 4. Vector magnetocardiograms of a 30-wk-old fetus with congenital atrioventricular block. The $\mathrm{p}$ waves are most clearly identified on the $\mathrm{Bz}$ tracing, revealing complete atrioventricular dissociation. The $\mathrm{T}$ waves are recognized on Bx tracing, although the amplitude is low. Signal-to-noise ratio of By tracing is unsatisfactory in this case. Accurate diagnosis would not be made in this case if By was the only signal measured. 
Table 2. FMCG diagnosis in fetuses with arrhythmias or structural heart disease

\begin{tabular}{|c|c|c|}
\hline $\begin{array}{c}\text { Case } \\
\text { no. }\end{array}$ & $\begin{array}{l}\text { Gestational age } \\
\quad(\text { wk-d) }\end{array}$ & $\begin{array}{l}\text { fMCG diagnosis } \\
\text { (ultrasound diagnosis) }\end{array}$ \\
\hline 1 & $22-0$ & Complete atrioventricular block \\
\hline 2 & $25-0$ & $\begin{array}{l}\text { n.p., normal PR interval (complete } \\
\text { ECD) }\end{array}$ \\
\hline 3 & $28-1$ & $\begin{array}{l}\text { n.p., normal PR interval (complete } \\
\text { ECD) }\end{array}$ \\
\hline 4 & $28-2$ & 2:1 Atrioventricular block \\
\hline 5 & $30-0$ & $\begin{array}{l}\text { Supraventricular premature } \\
\text { contraction }\end{array}$ \\
\hline 6 & $30-3$ & $\begin{array}{l}\text { Complete atrioventricular block, } \\
\text { short run ventricular premature } \\
\text { contraction }\end{array}$ \\
\hline 7 & $31-1$ & Wide QRS (TTS recipient) \\
\hline 8 & $31-1$ & Normal QRS (TTS donor) \\
\hline 9 & $32-2$ & $\begin{array}{l}\text { Normal } \mathrm{p} \text { and } \mathrm{PR} \text { interval (complete } \\
\mathrm{ECD} \text {, polysplenia) }\end{array}$ \\
\hline 10 & $33-0$ & $\begin{array}{l}\text { n.p. (ventricular septal defect, } \\
\text { possible } 18 \text { trisomy) }\end{array}$ \\
\hline 11 & $33-0$ & $\begin{array}{l}\text { 1:1 Supraventricular tachycardia, } \\
\text { long } \mathrm{RP}^{\prime}\end{array}$ \\
\hline 12 & $34-0$ & Long QT, 2:1 atrioventricular block \\
\hline 13 & $36-6$ & Ventricular premature contraction \\
\hline 14 & $37-2$ & High amplitude p waves (PA-IVS) \\
\hline 15 & $37-5$ & Ventricular premature contraction \\
\hline 16 & $38-4$ & $\begin{array}{l}\text { Supraventricular premature } \\
\text { contraction }\end{array}$ \\
\hline 17 & $39-2$ & $\begin{array}{l}\text { 2:1 Atrioventricular block, } \\
\text { ventricular premature contraction }\end{array}$ \\
\hline 18 & $39-2$ & $\begin{array}{l}\text { Supraventricular premature } \\
\text { contraction }\end{array}$ \\
\hline
\end{tabular}

ECD, endocardial cushion defect; n.p., nothing in particular; PA-IVS, pulmonary atresia with intact ventricular septum; TTS, twin-twin transfusion syndrome.

thermore, a 2:1 atrioventricular block associated with prolonged QT interval was diagnosed in a 34-wk-old fetus (Fig. 6).

\section{DISCUSSION}

In the present study, fetal PQRST waveforms obtained by composing the three magnetic components twice at different intervals showed similar configurations, and the differences in the time intervals (PR, QRS and VAT) between the first and the second measurements were smaller after composition (Bxyz) than before (Bx, By, Bz). Less fluctuation of the values of VAT and QRS interval suggests that the reproducibility of the waveform is acceptable. These results mean that the vector fMCG is much less affected by fetal presentation and that it is possible to standardize the fMCG waveform and establish the normal values of fetal cardiac time intervals. The PR interval in uncomplicated fetuses was rather constant during the period of concern and a little shorter than what we reported previously $(9,11)$. This is possibly due to the limited detection area covered by the four-channel SQUID system, resulting in failure of the sensors to register at those sites where the $p$ wave is the predominant feature. In fact, van Leeuwen $e t$ al. (12) demonstrated that the use of fewer channels led to a delayed setting of p-wave onset, resulting in shortening of the PR interval. On the other hand, significant prolongation of the QRS interval was noted with gestational age, a little longer than that recorded in our previous study $(9,11)$. This might have resulted from detection of low-amplitude depolarization at the onset and/or end of QRS through composition (12). The QT interval was similar to that reported in our previous reports $(9,11)$. In the present study, the success rate of recording of QRS and $\mathrm{p}$ waves was satisfactory. However, that for $\mathrm{T}$ wave was rather low, since higher detection rates for $\mathrm{T}$ wave, e.g. over than $90 \%$, have been recently reported $(13,14)$. This is partly because we excluded cases with ambiguous onset or endpoint of $\mathrm{T}$ wave as unsuccessful and because our study included a considerable number of fetuses at early stages of development (22\% of the fetuses were between 22 and $28 \mathrm{wk}$ of gestation) than the subjects in the above studies. In the fetuses that we considered successful for detecting $\mathrm{T}$ waves, the time resolution was improved by constructing a composite waveform.

For analysis of fetal arrhythmias, in which analysis of an unaveraged tracing is necessary, vector magnetometer also proved useful because at least one component of the vector magnetocardiogram showed efficient spatiotemporal resolu-

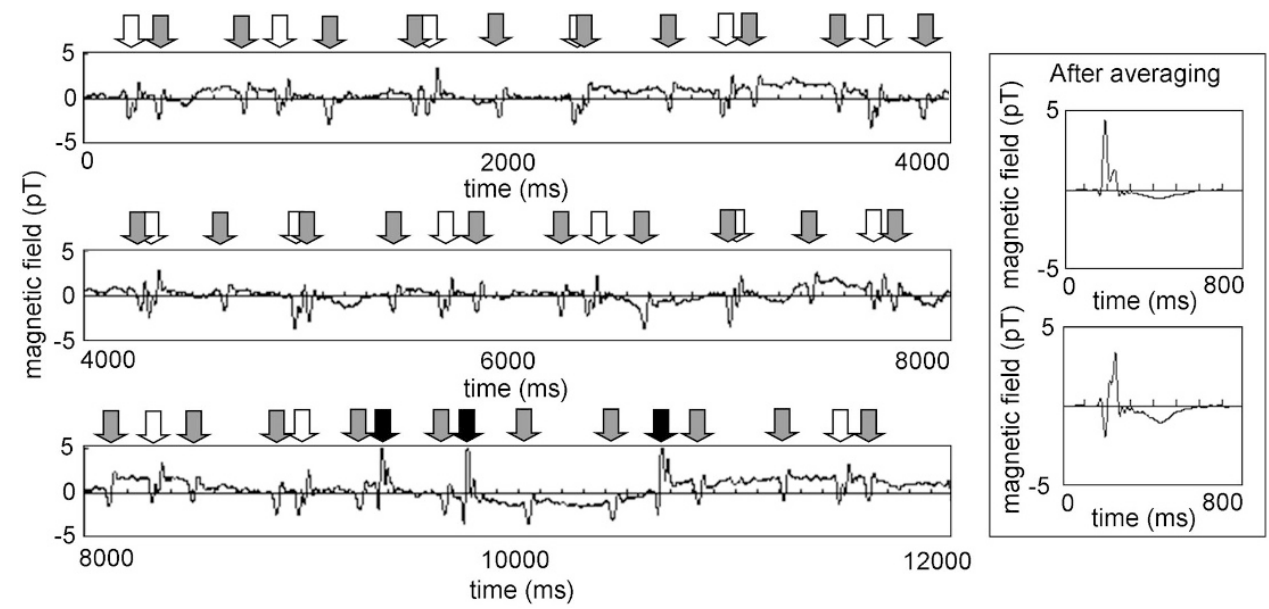

Figure 5. Vector magnetocardiograms of a 30-wk-old fetus with congenital atrioventricular block. Shaded arrows indicate p waves and white and black arrows indicate QRS complex. Two types of QRS waveforms are identified (right). The wider one (black arrows) appears in couplet manner with slight ST segment depression. 


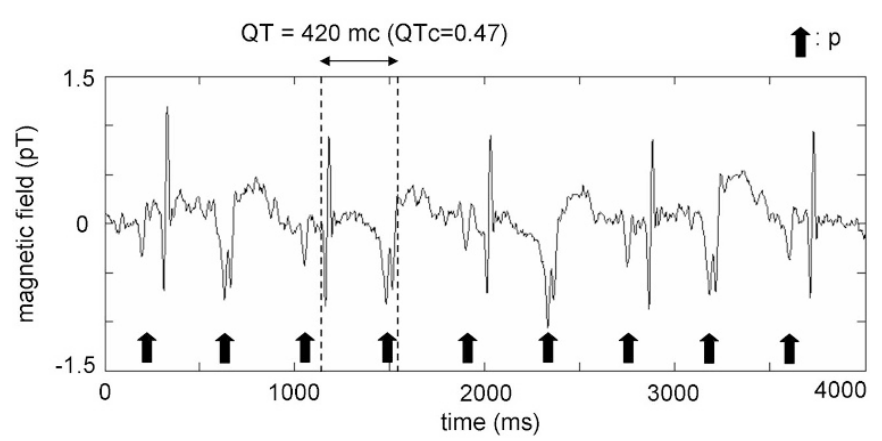

Figure 6. Vector magnetocardiograms of a 34-wk-old fetus with congenital 2:1 atrioventricular block, which had already been diagnosed by fetal echocardiography, but prolonged QT interval (QTc $=470 \mathrm{~ms}$ ) was first diagnosed by fetal magnetocardiography. The diagnosis was confirmed after birth by electrocardiography. The arrows indicate $\mathrm{p}$ waves, half of them being located at the peak of $\mathrm{T}$ waves.

tion regardless of fetal orientation or movement during measurement (Fig. 4). Thus, we can select a tracing with a high resolution among the waveforms of the three components for analysis even if the other channels show a low signal-to-noise ratio. Fetal arrhythmias have been analyzed by various methods. The M-mode echocardiography is the most commonly used method and has proved very useful at the first screening of fetal arrhythmia, but the time resolution is limited. Simultaneous Doppler sampling at two points such as at both superior vena cava and ascending aorta has made it possible to discriminate the type of fetal tachyarrhythmia (8). It is possible with fetal kinetocardiography using tissue Doppler echocardiography to estimate the origin of fetal arrhythmia (15). Although significant correlations between electrocardiographic and ultrasonographic values of cardiac time intervals have also been demonstrated $(16,17)$, these methods are all based on mechanical information and PQRST waveforms themselves cannot be obtained. On the other hand, fMCG shows time resolution and waveform configuration comparable with those of electrocardiography after birth. Wakai et al. (5) demonstrated that the use of fMCG allows determination of the mechanisms of initiation and termination of fetal supraventricular tachycardia as well as the type of arrhythmia. Since fMCG records the PQRST configurations, abnormal QRS shape, aberrant $\mathrm{p}$ wave in supraventricular tachycardia or QT prolongation can be demonstrated as in the present study. The fMCG demonstration of $F$ waves in atrial flutter, delta waves in preexcitation syndrome, and torsade de pointes in long QT syndrome have also been reported by other investigators $(3,7)$. The precise QT interval or ST segment deviation cannot be assessed by any method other than fMCG. In fact, several reports have demonstrated that congenital long QT syndrome can be diagnosed prenatally based on the accurate measurement of the QT interval $(18,19)$.

Recently, a European group proposed a standard method for data acquisition and data analysis to make fMCG a more definite and prevalent modality in prenatal diagnosis of fetal cardiac events (20). However, with a conventional SQUID system, the effects of fetal movement or orientation is inevi- table. In this regard, the vector $\mathrm{fMCG}$ technique could provide some solution for the limitation of this specialized field.

In conclusion, we have demonstrated in the present study that vector fMCG could provide standard fMCG waveforms as well as a complete and accurate reading of fetal cardiac events. Introduction of this methodology would enhance the use of fMCG as a reliable diagnostic tool in prenatal clinics.

\section{REFERENCES}

1. van Leeuwen P, Hailer B, Bader W, Geissler J, Trowitzsch E, Gronemeyer DH 1999 Magnetocardiography in the diagnosis of fetal arrhythmia. Br J Obstet Gynaecol 106:1200-1208

2. Kahler C, Grimm B, Schleussner E, Schneider A, Schneider U, Nowak H, Vogt L, Seewald HJ 2001 The application of fetal magnetocardiography (FMCG) to investigate fetal arrhythmias and congenital heart defects (CHD). Prenat Diagn 21:176182

3. Menendez T, Achenbach S, Beinder E, Hofbeck M, Klinghammer L, Singer H, Moshage W, Daniel WG 2001 Usefulness of magnetocardiography for the investigation of fetal arrhythmias. Am J Cardiol 88:334-336

4. Horigome H, Shiono J, Shigemitsu S, Asaka M, Matsui A, Kandori A, Miyashita T, Tsukada K 2001 Detection of cardiac hypertrophy in the fetus by approximation of the current dipole using magnetocardiography. Pediatr Res 50:242-245

5. Wakai RT, Strasburger JF, Li Z, Deal BJ, Gotteiner NL 2003 Magnetocardiographic rhythm patterns at initiation and termination of fetal supraventricular tachycardia. Circulation 107:307-312

6. Wakai RT, Wang M, Martin CB 1994 Spatiotemporal properties of the fetal magnetocardiogram. Am J Obstet Gynecol 170:770-776

7. Cuneo BF, Ovadia M, Strasburger JF, Zhao H, Petropulos T, Schneider J, Wakai RT 2003 Prenatal diagnosis and in utero treatment of torsades de pointes associated with congenital long QT syndrome. Am J Cardiol 91:1395-1398

8. Fouron JC, Fournier A, Proulx F, Lamarche J, Bigras JL, Boutin C, Brassard M, Gamache S 2003 Management of fetal tachyarrhythmia based on superior vena cava/aorta Doppler flow recordings. Heart 89:1211-1216

9. Stinstra J, Golbach E, van Leeuwen P, Lange S, Menendez T, Moshage W, Schleussner E, Kaehler C, Horigome H, Shigemitsu S, Peters MJ 2002 Multicentre study of fetal cardiac time intervals using magnetocardiography. $\mathrm{Br} \mathrm{J}$ Obstet Gynaecol 109:1235-1243

10. Kandori A, Miyashita T, Tsukada K, Horigome H, Asaka M, Shigemitsu S, Takahashi M, Terada Y, Mitsui T, Chiba Y 1999 A vector fetal magnetocardiogram system with high sensitivity. Rev Sci Instrum 70:4702-4705

11. Horigome H, Takahashi MI, Asaka M, Shigemitsu S, Kandori A, Tsukada K 2000 Magnetocardiographic determination of the developmental changes in PQ, QRS and QT intervals in the foetus. Acta Paediatr 89:64-67

12. van Leeuwen P, Lange S, Klein A, Geue D, Zhang Y, Krause HJ, Gronemeyer D 2004 Reproducibility and reliability of fetal cardiac time intervals using magnetocardiography. Physiol Meas 25: 539-552

13. van Leeuwen P, Lange S, Klein A, Geue D, Gronemeyer DH 2004 Dependency of magnetocardiographically determined fetal cardiac time intervals on gestational age, gender and postnatal biometrics in healthy pregnancies. BMC Pregnancy Childbirth 4:6

14. Grimm B, Kaehler C, Schleussner E, Schneider U, Haueisen J, Seewald HJ 2003 Influence of intrauterine growth restriction on cardiac time intervals evaluated by fetal magnetocardiography. Early Hum Dev 74:1-11

15. Rein AJ, O’Donnell C, Geva T, Nir A, Perles Z, Hashimoto I, Li XK, Sahn DJ 2002 Use of tissue velocity imaging in the diagnosis of fetal cardiac arrhythmias. Circulation 106:1827-1833

16. Dancea A, Fouron JC, Miro J, Skoll A, Lessard M 2000 Correlation between electrocardiographic and ultrasonographic time-intervals measurements in fetal lamb heart. Pediatr Res 47:324-328

17. Glickstein JS, Buyon J, Friedman D 2000 Pulsed Doppler echocardiographic assessment of the fetal PR interval. Am J Cardiol 86:236-239

18. Hamada H, Horigome H, Asaka M, Shigemitsu S, Mitsui T, Kubo T, Kandori A, Tsukada K 1999 Prenatal diagnosis of long QT syndrome using fetal magnetocardiography. Prenat Diagn 19:677-680

19. Hosono T, Kawamata K, Chiba Y, Kandori A, Tsukada K 2002 Prenatal diagnosis of long QT syndrome using magnetocardiography: a case report and review of the literature. Prenat Diagn 22:198-200

20. Grimm B, Haueisen J, Huotilainen M, Lange S, van Leeuwen P, Menendez T, Peters MJ, Schleussner E, Schneider U 2003 Recommended standards for fetal magnetocardiography. Pacing Clin Electrophysiol 26:2121-2126 